\title{
Ruidos articulares en estudiantes universitarios
}

\author{
Ana Cristina Mafla*, MsP., Diana Carolina Zambrano-Muñoz, Est. Odont., Yeimy Gómez-Díaz, \\ Est. Odont. ${ }_{1}$, Luz Andrea Dorado-Díaz, Est. Odont. ${ }_{1}$, Carlos Eduardo Bastidas-Eraso, Esp. ${ }_{1}$, Oswaldo \\ Esteban Chicaiza, Est. Odont. ${ }_{1}$
}

${ }_{1}$ Facultad de Odontología, Universidad Cooperativa de Colombia, Pasto, Colombia

Recibido: 12 de septiembre del 2014. Aprobado: 20 de octubre del 2014

*Autor de correspondencia: Ana Cristina Mafla Chamorro. Facultad de Odontología, Universidad Cooperativa de Colombia, sede Pasto, Colombia. Calle 18 n. $47-15$. Teléfonos: (57) 27314876 - 7313200 ext. 32. Correo electrónico: ana.mafla@campusucc.edu.co

Cómo citar este artículo: Mafla AC, Zambrano-Muñoz DC, Gómez-Díaz Y, Dorado-Díaz LA, Bastidas-Erazo CE, Chicaiza OE. Ruidos articulares en estudiantes universitarios. Rev Nac Odontol. 2014;10(19):35-46. doi: http://dx.doi.org/10.16925/od.v10i19.846

Resumen. Introducción: determinar la prevalencia de ruidos articulares en estudiantes universitarios de 20 años de edad en Pasto, Nariño, Colombia. Métodos: la muestra consistió de 173 individuos (77 hombres y 96 mujeres), quienes fueron evaluados de acuerdo con los Criterios Diagnósticos para la Investigación de Trastornos Temporomandibulares (RDC/TMD versión española) Eje I. Resultados: 36 individuos (20,8\%) tuvieron ruidos articulares; 32 (18,8\%) fueron clicking y 4 (2\%), crepitación. La razón mujer/hombre fue 1,7:1 ( $\mathrm{p}>0,05)$. Los individuos que estudiaron una carrera de ciencias de la salud presentaron una mayor frecuencia de ruidos articulares. En relación con las características de los ruidos articulares, 24 articulaciones temporomandibulares (ATM) tenían un/a clicking/crepitación a la apertura reproducible y 14 tenían un/a clicking/crepitación a la apertura recíproca. Además, 19 ATM presentaron un/a clicking/crepitación al cierre reproducible y 14 presentaron un/a clicking/crepitación al cierre recíproco. Dieciocho (50\%) estudiantes con ruidos articulares tenían una apertura limitada; 68 (39,3\%) estudiantes tenían dolor muscular o articular a la palpación; 17 individuos $(47,2 \%)$ con ruidos articulares tenían dolor muscular o articular a la palpación. Conclusión: estos resultados han mostrado las características clínicas de ruidos articulares en estudiantes universitarios. La prevalencia es similar a aquellas reportadas en otras poblaciones; existió una diferencia no significativa según el género, y casi la mitad de los individuos con ruidos articulares tenían dolor muscular o articular a la palpación. La presencia de clicking es un buen indicador de desplazamiento del disco con reducción.

Palabras clave: articulación temporomandibular, trastornos de la articulación temporomandibular, sonido, RDC/TMD, Colombia, epidemiología.

\section{Temporomandibular Joint Noise among University Students}

Abstract. Introduction: to determine the prevalence of joint noise among 20-year-old university students in Pasto, Nariño, Colombia. Methods: The sample consisted of 173 individuals (77 men and 96 women), who were evaluated according to Temporomandibular Disorder Research Diagnostic Criteria (RDC/TMD Spanish version) Axis. Results: 36 individuals $(20.8 \%)$ had joint noise; $32(18.8 \%)$ were clicking and $4(2 \%)$ had crepitation. The women/men ratio was 1.7:1 ( $p>0.05)$. Individuals studying a health science career had a higher joint noise rate. In relation to joint noise characteristics, 24 temporomandibular joints (тмJ) had clicking/crepitation at the reproducible aperture and 14 had clicking/crepitation at the reciprocal aperture. Additionally, 19 тмJ had clicking/crepitation at the reproducible closure and 14 had clicking/ crepitation at the reciprocal closure. $18(50 \%)$ students with joint noise had limited aperture; $68(39.3 \%)$ students had muscular or joint pain when palpated; 17 individuals (47.2\%) with joint noise had muscular or joint pain when palpated. Conclusion: The results show the clinical characteristics of joint noise among university students. The prevalence is similar to what is reported among other populations; there is an insignificant difference according to gender, and almost half of those with joint noise had muscular or joint pain when palpated. The presence of clicking is a good indicator of disk displacement with reduction.

Keywords: temporomandibular joint, temporomandibular joint disorders, sound, RDC/TMD, Colombia, epidemiology.

\section{Ruídos articulares em estudantes universitários}

Resumo. Introdução: odeterminar a prevalência de ruídos articulares em estudantes universitários de 20 anos de idade em Pasto, Nariño, Colômbia. Métodos: a mostra consistiu de 173 indivíduos (77 homens e 96 mulheres), que foram avaliados de acordo com os Critérios Diagnósticos para a Pesquisa de Transtornos Temporomandibulares (RDC/ TMD versão espanhola) Eixo I. Resultados: 36 indivíduos (20,8\%) tiveram ruídos articular; $32(18,8 \%)$ foram click e $4(2 \%)$, crepitação. A razão mulher/homem foi 1,7:1 ( $>$ > 0,05). Os indivíduos que estudaram uma faculdade de ciências da saúde apresentaram uma maior frequência de ruídos articulares. Em relação as características dos ruídos articulares, 24 articulações temporomandibulares (ATM) tinham um/a click/crepitação à abertura reproduzível 14 tinham um/a click/crepitação à abertura recíproco. Além disso, 19 ATM apresentaram um/a click/crepitação ao fechamento reproduzível e 14 apresentaram um/a click/crepitação ao fechamento recíproco. Dezoito (50\%) estudantes com ruídos articulares tinham uma abertura limitada; 68 (39,3\%) estudantes tinham dor muscular ou articular à palpação; 17 indivíduos $(47,2 \%)$ com ruídos articulares tinham dor muscular ou articular à palpação. Conclusão: estes resultados têm mostrado as características clínicas de ruídos articulares em estudantes universitários. A prevalência é similar àquelas reportadas em outras populações; existiu uma diferença não significativa segundo o gênero, e quase a metade dos indivíduos com ruídos articulares tinham dor muscular ou articular à palpação. A presença de click é um bom indicador de deslocamento do disco com redução.

Palavras-chave: articulação temporomandibular, transtornos da articulação temporomandibular, som, RDC/TMD, Colômbia, epidemiologia. 


\section{Introducción ${ }^{1}$}

La articulación temporomandibular (ATM) presenta diferentes estructuras anatómicas que permiten a la mandíbula hacer movimientos de rotación, lateralidad y traslación, que se relacionan con las funciones de masticación, habla y deglución. La ATM se diferencia de otras articulaciones porque tiene superficies articulares cubiertas con tejido fibroso avascular en lugar de cartílago hialino [1].

La Aтм es, por sus características, susceptible a diversas alteraciones. Se han reportado numerosas disfunciones de esta articulación en relación con trastornos músculo-esqueletales; una de las clasificaciones se refiere a problemas intra-articulares (intra-capsulares) y extraarticulares (extra-capsulares). En las primeras, se hace referencia a trastornos en disco articular, ligamentos del disco, membrana sinovial, fibrocartílago articular, y cóndilo mandibular y fosa glenoidea. En el segundo grupo, a músculo-esqueletales y a problemas del sistema nervioso central/sistema nervioso periférico [2].

Los trastornos internos de la ATM pueden manifestarse con dolor (que cuando es de la articulación, se denomina artralgia), y con sensación de espasmo cuando el paciente abre la boca. De la misma manera, pueden existir cambios en el movimiento del cóndilo/ disco, lo cual produce ruido articular que puede ir desde un clicking hasta una crepitación. La acción del cóndilo pasando bajo una banda posterior deformada, golpeando el disco y el componente temporal, ha sido sugerida como la causa más frecuente del ruido; también, el paso del cóndilo más allá del tubérculo articular y la perforación del disco. Sin embargo, la crepitación ha sido considerada como un indicativo de enfermedad articular avanzada como la artritis degenerativa [3].

Los signos clínicos que podrían ser indicativos de trastornos internos de la ATM que corresponden a ruidos articulares se establecen en $25 \%$ como clickings y en $15 \%$ como crépitus [4]. Los resultados de la investigación de John et al. [5] en 3033 individuos entre 10 y 18 años mostraron que $14,7 \%$ presentaba ruidos articulares autorreportados.

De la misma forma, el 14,3\% y el 12,3\% de mujeres y hombres, respectivamente, presentaron ruido articular en el estudio de Feteih [6], en 385 adolescentes de 12 a 16 años en Arabia Saudita. En el primer reporte de un estudio de seguimiento a 20 años por Egermark

1 Este estudio fue apoyado por el Comité para el Desarrollo de la Investigación (CONADI), Universidad Cooperativa de Colombia. et al. [7], en Suecia, se observó que en edades de 17 a 25 años la presencia de ruido articular frecuente era de $8 \%$. Al final de esta investigación, Magnusson et al. [8], muestran los cambios existentes desde edades de 7 a 15 años, y en los que no había ruidos articulares frecuentes, aparecen en $5 \%$ cinco años después, se incrementa a $8 \% 10$ años después y este porcentaje se mantiene a los 20 años de seguimiento.

Se han establecido como factores de riesgo enfermedades por proximidad como otitis externa o media, mastoiditis, parotiditis, rinosinusopatías, o de tipo dermatológico, odontológico y oftalmológico; infecciones sistémicas como sífilis, tuberculosis, fiebre tifoidea, neumonía o fiebre reumática (streptococcus beta-hemolítico); enfermedades sistémicas como procesos tumorales, artritis reumatoidea, fibromialgia, espondilitis, esclerosis múltiple, hiperuricemia, psoriasis, vasculitis, artritis.

De igual manera, alteraciones intra-articulares como las del complejo cóndilo-disco; desplazamiento discal anterior; luxación con reducción y luxación sin reducción; incompatibilidades articulares estructurales; subluxaciones, sinovitis, capsulitis; y por traumatismos, entre ellos, macro como golpes directos o micro como bruxismo; y enfermedades psiquiátricas como tensión emocional, neurosis [9] y tipo de personalidad [10], pueden desarrollar trastornos de la ATM.

Entre los factores de riesgo de los trastornos temporomandibulares relacionados con la cavidad bucal, están el bruxismo y el rechinamiento asociados con dolor miofacial (oR $=4,9$ IC al 95\% 3,0-7,8), en especial la presencia de dolor miofacial en mujeres ( $\mathrm{OR}=3,8$ IC al 95\% 2,4-6,1); y el desplazamiento del disco (oR $=2,5$ IC al 95\% 1,4-4,3), el cual disminuye con la edad (11 a 24 años con OR $=5,6$ IC al 95\% 2,7-11,2; de 25 y 37 años con oR = 2,0 IC al 95\% 1,0-4,0; estos valores fueron mayores en los sujetos de 38 y 79 años) [11].

También se ha asociado a los arcos dentales cortos con cambios en la articulación temporomandibular [12]. En relación, específicamente, con los ruidos articulares, se reporta que estos aumentan de modo significativo en individuos que habían perdido más dientes. Sin embargo, se menciona que no hay una diferencia clínica significativa entre individuos con arcos cortos de 3 a 5 unidades oclusales y aquellos con unos arcos completos en términos de signos y síntomas de desórdenes temporomandibulares [13], y se han observado asociaciones positivas entre la mordida cruzada unilateral y los ruidos articulares. Los resultados sugieren que la mordida cruzada incrementa el 
riesgo del desplazamiento del disco con reducción [14], aunque otros estudios no lo relacionan [15].

Muchos individuos presentan ruidos articulares como resultado de movimientos mandibulares disfuncionales espasmódicos, algunas veces con daño anatómico reconocible a las estructuras articulares. Estos ruidos pueden aparecer y desaparecer con frecuencia y recidivar después del tratamiento funcional, especialmente en personas jóvenes [16]. También, se ha informado que los adultos con ruidos articulares tienen un maxilar de acuerdo con el plano sagital más corto y una mandíbula diagonal; además, cuando presentan ruidos tipo crepitación, presentan una base de cráneo anterior y posterior y la faringe más corta. Es decir, estos signos reportados de los trastornos temporomandibulares están asociados con un tercio medio de la cara en el plano sagital más corto [17]. Por esta razón, es importante controlar la aparición y permanencia de los ruidos articulares, porque puede ser una condición que podría ser evitable.

Debido a lo anterior, el objetivo de este proyecto es evaluar la presencia de ruidos articulares con los Criterios Diagnósticos para la Investigación de Trastornos Temporomandibulares (RDC/TMD) en universitarios de 20 años de San Juan de Pasto (Nariño, Colombia), a través de un estudio descriptivo-transversal que nos ayudará a tener un mejor entendimiento sobre la enfermedad y a formular nuevas hipótesis de estudio en estos jóvenes, con el propósito de prevenir la aparición de trastornos de la ATM en este grupo susceptible.

\section{Métodos}

Se diseñó un estudio descriptivo-transversal de febrero a noviembre de 2010, cuya aplicación fue realizada previa aprobación del Comité de Ética de Ciencias de la Salud de la Universidad Cooperativa de Colombia, Pasto (Acta N. ${ }^{\circ}$ CECSO1-09). La muestra estuvo conformada por 173 individuos de 20 años que de manera voluntaria aceptaron a la evaluación en diferentes facultades de la Universidad Cooperativa de Colombia, sede Pasto, de la Universidad Mariana, de la Institución Universitaria CESMAG y de la Universidad de Nariño.

Se incluyeron jóvenes con y sin cualquier tipo de ruido articular, yjóvenes sintomáticos o asintomáticos a la palpación de la ATM. En relación con los criterios de exclusión, no se evaluó a jóvenes con enfermedades por proximidad, dermatológicas, con alteraciones intraarticulares previas, traumatismos o con compromiso sistémico. Para la recolección de datos, se diseñó un formato de recolección basado en los Criterios Diagnósticos para la Investigación de Trastornos Temporomandibulares (RDC/TMD versión española) Eje I [18], para el registro de ruidos articulares y variables relacionadas con ellos, como lo son los movimientos de apertura y cierre y la presencia de dolor.

\section{Procedimiento}

1) La muestra se obtuvo a través de las bases de datos institucionales y fue editada según edad cumplida, que para el estudio fue 20 años, y según el programa y el semestre al cual pertenecían.

2) El trabajo de campo se llevó a cabo en los diferentes programas de la Universidad Cooperativa de Colombia, sede Pasto, de la Universidad Mariana, de la Institución Universitaria CESMAG y de la Universidad de Nariño. Se inició la evaluación con los estudiantes según los horarios de estudio y la disponibilidad de los investigadores previo consentimiento informado.

3) Dos investigadores hicieron el diagnóstico de ruidos articulares previa calibración. Se solicitó al estudiante que se sentara en una angulación de $90^{\circ}$, y se evaluó bi-manualmente y con un Pie de Rey marca L\&W Tools Guaranted Quality Verter Caliper (0-150x0,05 mm/0,6'x1/126'), de la siguiente manera a través de palpación:

Palpación de ruidos articulares durante el movimiento vertical

1) El investigador indicó la presencia o ausencia de ruidos; si estaban presentes, anotó el tipo de ruido apreciado.

2) Se colocó el dedo índice izquierdo sobre la articulación derecha y el derecho sobre la izquierda (área preauricular). La yema del dedo derecho se colocó anterior al tragus de la oreja.

3) Se pidió al estudiante que abra lentamente lo más que le sea posible, incluso si ello le causa dolor. Al final de cada cierre, el estudiante puso los dientes en contacto en una posición de máxima intercuspidación.

4) Se le dijo al estudiante: "Mientras yo tenga mis dedos sobre su articulación, le pediré que abra su boca lentamente lo más que pueda y después, que cierre su boca lentamente hasta que sus dientes estén completamente juntos". 
5) Se le pidió al estudiante que abra y cierre la boca tres veces. Se anotó el sonido que la articulación había producido en apertura o cierre tal como es detectado durante la palpación, de acuerdo con los siguientes parámetros:

\section{- Definición de ruidos articulares}

Se definió como clicking un sonido preciso, de corta y limitada duración con un claro comienzo y final, el cual generalmente suena como "clic", y se registró solo si el clicking ocurría en dos de tres movimientos de apertura y cierre. La crepitación gruesa se determinó como un sonido continuo, en un periodo largo durante el movimiento mandibular. No es breve como el clicking o el pop: el sonido se puede apreciar como un ruido superpuesto continuo, que no es tenue, es como el ruido de hueso sobre hueso o como moliendo una piedra contra otra piedra.

La crepitación fina se describió como un sonido rechinante fino que es continuo en un periodo más largo durante el movimiento mandibular de apertura o cierre. No es breve como el clicking: el sonido puede apreciarse como un ruido sobrepuesto continuo; puede ser descrito como un sonido de frotamiento o crujido sobre una superficie áspera.

\section{- Evaluación de clicking}

Se estableció como clicking reproducible en movimiento de apertura si durante los movimientos de apertura o cierre desde la posición de máxima intercuspidación, un clicking fue notado en dos o tres movimientos de apertura; y se determinó como clicking reproducible en movimiento de cierre a un clicking presente en dos o tres movimientos mandibulares de cierre. Se definió un clicking recíproco reproducible como la presencia de un ruido en apertura y cierre y se midió con una regla milimetrada durante estos movimientos. La eliminación de los dos clickings se determinó cuando el sujeto abrió y cerró la boca en protrusión. Si el clicking cesaba, el análisis indicaba que no es un clicking recíproco: aunque un clicking ha estado presente, este no fue constante.

\section{- Evaluación de apertura y presencia de dolor}

Apertura (mandibular) no asistida sin dolor: se solicitó al estudiante que abra la boca lo más que pueda (no asistido) sin que sienta ningún dolor, se calculó la medida con el Pie de Rey desde el borde incisal del incisivo central superior que esté más vertical, y se midió la distancia entre este y el borde incisal del incisivo central inferior. Si los dos incisivos tenían la misma longitud vertical, se tomó la medición entre 21 y 31 ; si la apertura era menor de $30 \mathrm{~mm}$, se pidió al estudiante repetir la apertura. Si la segunda vez la medida era menor de $30 \mathrm{~mm}$, entonces se la registraba.

Apertura (mandibular) máxima no asistida: se indicó al estudiante que relaje la mandíbula y luego que abra la boca lo más que pueda, aunque sienta dolor; se hizo la medición con el instrumento entre el borde incisal del incisivo central superior que esté más vertical, y se midió la distancia entre este y el borde incisal del incisivo inferior. Se preguntó al estudiante si tenía dolor y su localización exacta. La apertura fue clasificada de 35 a $50 \mathrm{~mm}$ como normal, menor a $35 \mathrm{~mm}$ como disminuida y mayor a $50 \mathrm{~mm}$ como hipermetría.

Apertura (mandibular) máxima asistida: se pidió al estudiante que abra la boca lo más que pueda, aunque sienta dolor. Una vez que tuvo la boca abierta, se puso el pulgar contra el borde de los incisivos superiores, y en forma cruzada, el índice sobre los incisivos centrales mandibulares. En esta posición se obtuvo la palanca necesaria para forzar una apertura mandibular mayor, y se usó presión moderada, sin forzar la apertura. Con el Pie de Rey, se midió verticalmente desde el borde incisal del incisivo central superior de referencia al borde incisal del incisivo inferior. Se registró si existió dolor y su localización.

Sobremordida vertical: se solicitó al estudiante que cierre la boca y que mantenga los dientes completamente juntos. Con un lápiz se marcó una línea en el punto donde llega el borde del incisivo central superior de referencia al incisivo inferior. Se midió la distancia entre el borde incisal del incisivo inferior marcado y la línea realizada.

- Dolor articular a la palpación: descripción de sitios específicos de palpación articular (una libra de presión digital)

Polo lateral: se colocó el dedo índice justo antes del tragus de la oreja y sobre la ATM del estudiante, y se le pidió que abra ligeramente la boca hasta sentir la traslación del polo del cóndilo hacia adelante. Se usó una libra de presión en el lado que se está palpando y se le sostuvo la cabeza con la mano opuesta. 
Inserción posterior: se colocó el dedo meñique derecho en el meato izquierdo del estudiante y el dedo meñique izquierdo en el meato derecho. Se apuntó la yema de los dedos hacia el examinador y se le indicó al sujeto que abra ligeramente la boca (o ampliamente si era necesario), para asegurarse de que se pudiera sentir el movimiento de la articulación con la punta de los dedos. Se presionó firmemente primero un lado y luego el otro, mientras los dientes del estudiante estaban completamente juntos.

\section{Variabilidad intra e interobservador}

La variabilidad se registró en las diferentes universidades, según los casos encontrados de acuerdo con el Índice de Kappa de Cohen de máxima probabilidad de acierto. Cada evaluador observó 18 casos dos veces durante las diferentes sesiones, que consistían en el registro de 5 a 8 personas. En el momento en que un individuo con ruido articular era detectado, se lo dejaba de último para que lo volviera a evaluar el mismo examinador y otro diferente.

La variabilidad intraobservador del primer evaluador fue de 0,77 y del segundo, de 0,83. Los dos evaluadores examinaron a los 36 sujetos, y la variabilidad inter-observador fue de 0,86. En algunos casos, el diagnóstico fue confirmado por el paciente para determinar la variabilidad, debido a que algunos ruidos articulares no eran reproducibles y en el momento de la nueva evaluación no se presentaron.

\section{Análisis de la información}

Por medio del paquete estadístico sPss versión 17 , se creó una base de datos en la que se registraron medidas descriptivas, como frecuencias y porcentajes, y medidas como Chi, para comparación de variables demográficas y específicas, como las relacionadas con apertura y la presencia de dolor. Los resultados se organizaron en tablas.

\section{Resultados}

\section{Características de la muestra}

Existió un mayor número de mujeres (55,5\%), y los estratos predominantes fueron el 3 y el 4 (52,6\%), principalmente de la Institución Universitaria CESMAG
$(42,2 \%)$ y del programa de ciencias de la salud (37\%). En la tabla 1, se describen las características demográficas de la población de estudio.

Tabla 1. Características sociodemográficas de 173 jóvenes universitarios de 20 años de San Juan de Pasto (Nariño, Colombia)

\begin{tabular}{|l|c|c|}
\hline \multicolumn{1}{|c|}{ Características sociodemográficas } & \multicolumn{2}{c|}{$\begin{array}{c}\text { Estudiantes } \\
\text { universitarios }\end{array}$} \\
\hline Sexo & & \\
\hline Masculino & 77 & $44,5 \%$ \\
\hline Femenino & & $55,5 \%$ \\
\hline Estrato & 82 & $47,4 \%$ \\
\hline Estratos 1 y 2 & 91 & $52,6 \%$ \\
\hline Estratos 3 y & - & \\
\hline Estrato 5 & & \\
\hline Universidad & 60 & $34,7 \%$ \\
\hline $\begin{array}{l}\text { Universidad Cooperativa de Colombia, } \\
\text { Pasto }\end{array}$ & 8 & $18,5 \%$ \\
\hline Universidad Mariana & 32 & $42,2 \%$ \\
\hline Institución Educativa CESMAG & 73 & $4,6 \%$ \\
\hline Universidad de Nariño & & \\
\hline Programa & 64 & $37,0 \%$ \\
\hline Ciencias de la salud & 47 & $27,2 \%$ \\
\hline Ciencias sociales aplicadas & 43 & $24,9 \%$ \\
\hline Ciencias humanas & 12 & $6,9 \%$ \\
\hline Ingenierías & 5 & $2,9 \%$ \\
\hline Lingüística, letras y arte & 2 & $1,2 \%$ \\
\hline Ciencias agrarias & & \\
\hline & & \\
\hline
\end{tabular}

Fuente: elaboración propia

La prevalencia de ruidos articulares fue de 36 $(20,8 \%)$, de los cuales $32(18,8 \%)$ eran tipo clicking y 4 (2\%) eran tipo crepitación gruesa. Del grupo de personas con ruidos articulares, se observaron $13(36,1 \%)$ casos en hombres y $23(63,9 \%)$ en mujeres $(p>0,05)$; la proporción observada en esta variable en el grupo de hombres y mujeres fue $16,8 \%$ y $23,9 \%$, respectivamente. La razón entre sexo mujer/hombre fue de 1,7:1.

Según los estratos, $12(33,3 \%)$ estudiantes de estrato 1 y 2 presentaron ruido articular y $24(66,7 \%)$ fueron de estrato 3 y $4(p=0,05)$. Los ruidos articulares se observaron en la Universidad Cooperativa de Colombia, sede Pasto con 17 (47,2\%) estudiantes, seguido de la Institución Educativa Cesmag con 9 (25,0\%) $(p>0,05)$. Sin embargo, de acuerdo con el número de estudiantes evaluados en cada una de las universidades la presencia de ruidos osciló entre 12 y $28 \%$. Según el programa, $19(52,8 \%)$ ruidos articulares se presentaron 
en los programas de ciencias de la salud, seguido de ciencias sociales aplicadas y ciencias humanas, ambas con 7 casos $(19,4 \%)(p>0,05)$.

De los 36 estudiantes con ruido articular, 8 $(22,2 \%)$ tenían tratamiento ortodóncico y $3(8,3 \%)$ habían sufrido un trauma leve, quienes fueron de sexo masculino. En la tabla 2, se exponen la distribución y las características de los ruidos articulares según reproducibilidad y reciprocidad. El mayor número de ruidos articulares se observó en apertura y en el lado izquierdo, con 17 casos $(47,2 \%)$ tipo clicking y uno $(2,7 \%)$ tipo crepitación gruesa. Los ruidos articulares en apertura se presentaron a los 22,83 $\mathrm{mm} \pm 9,57 \mathrm{~mm}$, $\mathrm{y}$ en cierre, a los $11,28 \mathrm{~mm} \pm 11,20 \mathrm{~mm}$. De acuerdo con la ubicación, el ruido articular clicking/crepitación se observó a los 20,20 $\mathrm{mm} \pm 7,76 \mathrm{~mm}$ en el derecho y en el izquierdo a los $23,76 \mathrm{~mm} \pm 11,65 \mathrm{~mm}$.

Tabla 2. Distribución y características de ruidos articulares en apertura y cierre por localización, en 36 estudiantes

\begin{tabular}{|l|c|c|c|}
\hline \multicolumn{1}{|c|}{ Características clínicas } & Clicking F (\%) & Crepitación F (\%) & Total F (\%) \\
\hline Ruido articular & & $1(2,7 \%)$ & $16(44,3 \%)$ \\
\hline Apertura derecha & $15(41,6 \%)$ & $1(2,7 \%)$ & $12(33,2 \%)$ \\
\hline Cierre derecho & $11(30,5 \%)$ & $2(5,4 \%)$ & $19(52,6 \%)$ \\
\hline Apertura izquierda & $17(47,2 \%)$ & - & $9(25,0 \%)$ \\
\hline Cierre izquierdo & $9(25,0 \%)$ & $9(25,0 \%)$ \\
\hline Reproducibilidad & & & $10(27,7 \%)$ \\
\hline Apertura derecha & & & $13(36,1 \%)$ \\
\hline Cierre derecho & & & $9(25,0 \%)$ \\
\hline Apertura izquierda & & & $8(22,2 \%)$ \\
\hline Cierre izquierdo & & & $6(16,6 \%)$ \\
\hline Reciprocidad apertura/cierre & & & \\
\hline Derecha & & & \\
\hline Izquierdo & & & \\
\hline
\end{tabular}

Fuente: elaboración propia

Para la evaluación de apertura maxilar, la medición de los dientes fue principalmente en 164 (94,9\%) casos entre 21 y $31 \mathrm{~mm}$, y solo en $9(5,2 \%)$ casos fue entre 11 y $41 \mathrm{~mm}$. La apertura máxima no asistida sin dolor de los individuos fue de $35,29 \mathrm{~mm}$ y \pm 7,55 mm; la apertura mandibular máxima no asistida con o sin dolor fue de $42,58 \mathrm{~mm} \pm 7,45 \mathrm{~mm}$, y la media de la apertura mandibular máxima asistida fue de $46,36 \mathrm{~mm}$ $\pm 6,76 \mathrm{~mm}$.

Entre los estudiantes, $78(45,1 \%)$ presentaron una apertura disminuida; 90 (52\%) tenían una apertura normal, y $5(2,9 \%)$, una apertura aumentada. De los estudiantes con ruido articular, 18 (50\%) tenían una apertura limitada. En general, 68 (39,3\%) presentaron dolor, y entre los estudiantes que tenían ruido articular, $17(47,2 \%)$ tenían sintomatología dolorosa. En apertura máxima no asistida, $65(37,5 \%)$ referían dolor, mientras $97(56,1 \%)$ estudiantes en apertura asistida lo tenían.

En la tabla 3, se observa la discriminación del dolor según aperturas y ubicación. Se observó que el mayor porcentaje de este síntoma estuvo en apertura mandibular asistida. En la apertura mandibular no asistida, $2(5,6 \%)$ de los estudiantes con ruido articular presentaron dolor muscular y $12(33,3 \%)$ tenían dolor articular $(p<0,05)$. En la apertura mandibular asistida, seis $(16,7 \%)$ de los estudiantes con ruido articular tenían dolor muscular, mientras que $16(44,4 \%)$ $(p<0,05)$ tenían dolor articular. La sobremordida vertical fue de $1,94 \mathrm{~mm} \pm 1,42 \mathrm{~mm}$. Los casos que tuvieron una sobremordida entre 1 y $3 \mathrm{~mm}$ fueron $116(67,1 \%)$, y $57(32,9 \%)$ estudiantes tuvieron valores mayores a $3 \mathrm{~mm}$ y situaciones de relación inversa o borde a borde. 
Tabla 3. Dolor muscular y articular en aperturas no asistida $y$ asistida en 173 estudiantes

\begin{tabular}{|l|c|c|c|c|}
\hline \multicolumn{1}{|c|}{ Apertura } & \multicolumn{2}{|c|}{ No asistida } & \multicolumn{2}{c|}{ Asistida } \\
\hline & F & $\%$ & F & $\%$ \\
\hline Dolor muscular & & & & \\
\hline Derecho & 12 & $18,4 \%$ & 18 & $18,6 \%$ \\
\hline Izquierdo & 6 & $9,2 \%$ & 15 & $15,4 \%$ \\
\hline Dolor articular & & & & \\
\hline Derecho & 7 & $10,7 \%$ & 9 & $9,3 \%$ \\
\hline Izquierdo & 8 & $12,3 \%$ & 13 & $13,4 \%$ \\
\hline $\begin{array}{l}\text { Dolor muscular derecho e } \\
\text { izquierdo }\end{array}$ & 16 & $24,7 \%$ & 23 & $23,7 \%$ \\
\hline $\begin{array}{l}\text { Dolor articular derecho e } \\
\text { izquierdo }\end{array}$ & 16 & $24,7 \%$ & 19 & $19,6 \%$ \\
\hline Total & 65 & $100 \%$ & 97 & $100 \%$ \\
\hline
\end{tabular}

Fuente: elaboración propia

En relación con la presencia de dolor en el polo lateral externo y la inserción posterior de la articulación temporomandibular, 19 (11\%) estudiantes presentaron dolor en el lado derecho en cada uno de estos dos sitios, 18 (10,4\%) tenían dolor en el lado izquierdo y 9 (5,2\%), en el derecho e izquierdo del polo lateral externo. En relación con la presencia de dolor en la inserción posterior (canal auditivo), 10 (5,8\%) lo tenían en el lado izquierdo y 6 (3,5\%), en el derecho e izquierdo. De las 36 personas con ruido articular, $11(30,6 \%)$ tenían dolor en el polo lateral externo y 9 (25\%) lo tenían en la inserción posterior (canal auditivo). En la tabla 4, se especifica la respectiva ubicación.

Tabla 4. Dolor articular a la palpación en el polo lateral externo y la inserción posterior (canal auditivo) de 36 estudiantes con ruido articular

\begin{tabular}{|l|c|c|c|c|}
\hline \multirow{2}{*}{ Ruido articular } & \multicolumn{2}{|c|}{ Polo lateral externo } & \multicolumn{2}{c|}{$\begin{array}{c}\text { Inserción posterior } \\
\text { (canal auditivo) }\end{array}$} \\
\hline & $\mathrm{F}$ & $\%$ & $\mathrm{~F}$ & $\%$ \\
\hline Sin dolor & 25 & $69,4 \%$ & 27 & $75 \%$ \\
\hline Dolor & & & & \\
\hline Derecho & 4 & $11,1 \%$ & 5 & $13,9 \%$ \\
\hline Izquierdo & 5 & $13,9 \%$ & 3 & $8,4 \%$ \\
\hline $\begin{array}{l}\text { Dolor derecho e } \\
\text { izquierdo }\end{array}$ & 2 & $5,6 \%$ & 1 & $2,7 \%$ \\
\hline Total & 36 & $100 \%$ & 36 & $100 \%$ \\
\hline
\end{tabular}

Fuente: elaboración propia

\section{Discusión}

El 20,8\% de los jóvenes universitarios de la ciudad de Pasto (Nariño, Colombia) presentó ruidos articulares, de los cuales el $18,8 \%$ fue tipo clicking y el $2 \%$ fue crepitación. En el reporte de Uhac et al. [19], en 100 individuos de Croacia se observó la presencia de ruido articular tipo clicking en $28 \%$ y de crepitación en $2 \%$. Una investigación realizada por Soto et al. [20], en Cali (Colombia) muestra, a través del índice de Helkimo en edades de 5 a 14 años, una prevalencia de ruidos articulares en dentición mixta de 5,8\% y permanente de $2,4 \%$ en apertura.

La presencia o ausencia de ruidos articulares proporciona información sobre la ubicación del disco, aunque la ausencia de ruidos no significa que la posición del disco esté en una situación normal. En un estudio se observó que el 15\% de las articulaciones asintomáticas silenciosas presentaban desplazamientos discales en las artrografías [21]. Hay dos mecanismos básicos que pueden explicar la ocurrencia de ruidos en la ATM. El clic puede ser la consecuencia de una relación cóndilodisco alterada, que ocurre debido al daño de las fibras del ligamento posterior, i.e. hiperfunción del vientre superior unilateral del músculo pterigoideo lateral. Por su parte, la crepitación es usualmente resultado de las superficies irregulares de la articulación, la mayoría debido frecuentemente a cambios degenerativos.

A su vez, existió una diferencia entre el ruido articular presente en mujeres y hombres, con una razón de 1,7:1, aunque no fue estadísticamente significativa como lo muestran otros estudios relacionados con trastornos temporomandibulares (TTM) [22, 23]. En general, las mujeres tienen menos fuerza muscular que los hombres. Además, el sistema masticatorio de las mujeres puede tener menos habilidad para resistir estimulaciones nocivas de oclusión anormal, por lo cual las mujeres podrían ser más susceptibles a los TTM que los hombres [24].

Sin embargo, la explicación de que no existiera una diferencia significativa en este caso, es porque la etiología de algunos ruidos en los hombres fue el haber sufrido un trauma leve. El papel del trauma podría ser controversial en la etiología de los TTM, pero se ha reportado que contusiones en cabeza y cuello son consideradas como factores de riesgo significativos para desarrollar alguno de estos trastornos, puesto que pueden producir desarreglos internos de la ATM [25]. Por otro lado, la presencia de ruido articular en estudiantes de sexo masculino fue $16,8 \%$, porcentaje 
levemente superior a lo observado por Choi et al. [26] en jóvenes coreanos de 19 años, que fue 14,3\%.

Durante los últimos 15 años, se ha visto un marcado incremento en la investigación sobre la naturaleza de la relación entre el estrato socioeconómico (ESE) y la salud. Las investigaciones iniciales a este respecto mostraron un grado de asociación, no solo para el caso de aquellos que están en la pobreza, sino también para todos los demás niveles de ESE [27].

En la presente investigación, se observó que el $66,7 \%$ de los individuos con ruidos articulares pertenecían al estrato 3 y 4 , que corresponde a una clase media. Las diferencias observadas en relación con la salud entre una clase trabajadora y una clase media, se deben a que en la primera hay una tendencia a somatizar los problemas, mientras en la segunda muchos de los signos y síntomas tienden a reportarse más [28]. Por otra parte, se han relacionado los niveles de cortisol sérico, la secreción de esteroides gonadales y la función inmune con el rango social en modelos animales [29], y el primero con TTM.

Los resultados indican que posiblemente existen diferentes estresores en los estratos. Quizá, en este caso en particular, la asociación del estrato medio con la presencia de ruido articular era debido a que muchos de los estudiantes de Odontología y Medicina pertenecían a este estrato y fueron los que presentaron mayor frecuencia de ruido articular, como se explica en el próximo párrafo. También, otra condición es que los individuos de estratos inferiores se enfocan más en solventar sus necesidades básicas, mientras que en los más altos la atención se podría dirigir a mantener su nivel de vida.

Las profesiones de la salud, especialmente Odontología y Medicina, se han reportado como estresantes. En este sentido, la naturaleza estresante de la profesión odontológica está bien establecida, porque se ha afirmado que es una de las más desafiantes y con campos de estudio exigentes, puesto que se espera que sus estudiantes adquieran competencias académicas y clínicas, y habilidades interpersonales. Además, los altos niveles de estrés percibido en estudiantes de Odontología se han asociado con signos y síntomas físicos, estrés psicológico, cansancio emocional, entre otros [30].

En la carrera de Medicina, las situaciones estresantes en estudiantes se han relacionado con síntomas de depresión, deficiencias en el desempeño en los salones de clase y en las prácticas clínicas, y también pueden conducir a problemas de salud [31].
A su vez, otros espacios asociados a las ciencias de la salud informan una alta frecuencia de alteraciones como ruido articular. Tanto así que, en un estudio realizado por Larrucea et al. [32], con funcionarios del hospital y de la Universidad de Talca, en Chile, de edades entre 21 y 50 años, se observó que el 48,5\% tenía ruido articular autorreportado en apertura y/o cierre, a diferencia de otros [33].

Por su parte, el 22,2\% de los estudiantes con ruido articular tenían ortodoncia, cuya relación con la presencia de dicha alteración ha sido controversial. Este tipo de trastorno puede ser causado por condiciones como retrusión natural de la mandíbula, mordida cruzada posterior, desarmonía maxilomandibular o interferencias oclusales, que pudieron existir previamente al tratamiento [34]. Sin embargo, el estudio de Akana et al. [35], a través de la medición de vibraciones de los ruidos articulares, demostró que estos disminuyeron en pacientes que presentaron mordida cruzada lateral, mientras que aumentaron en pacientes con clase II división I, cuando estuvieron en tratamiento ortodóncico. Para cualquiera de los casos, se sugiere una evaluación clínica de la ATM previa y posterior a este tipo de tratamiento bajo una guía clínica estandarizada. Otras condiciones relacionadas con oclusión muestran que en 521 individuos, quienes habían perdido de 1 a 14 dientes, hubo una asociación significativa entre la pérdida de los primeros premolares del maxilar y la presencia de la disfunción de la ATM, y que no existe una explicación etiológica para este resultado [36].

En relación con las características de la presencia de ruido articular, los resultados muestran que el mayor número de casos $(47,2 \%)$ tuvo lugar en apertura y en el lado izquierdo. Una de las posibles causas es la sobrecarga masticatoria que puede ocurrir en ese lado, debido a la tendencia de usar más un lado del cuerpo que otro. En el caso de la boca, implica la ingesta de alimentos teniendo en cuenta los patrones de lateralidad de las manos, y al ser diestra la mayoría de población, dicha tendencia sería la sobrecarga masticatoria en el lado izquierdo, condición que se ha expresado en otros campos de la Odontología como la colocación de las comisuras labiales en los movimientos de la sonrisa natural [37]. De la misma manera, la efectividad masticatoria se ha asociado a trastornos de la ATM y otra explicación podría ser el desarrollo muscular determinado también por la lateralidad, al igual que la presión ejercida en el lado escogido al dormir, condiciones que también deberían ser estudiadas. 
El análisis de los ruidos de la ATM en movimientos mandibulares es un indicativo de la severidad de alteraciones internas de la ATM. El clicking y la crepitación pueden ser signos de desórdenes de la articulación; el primero indica un desplazamiento del disco con reducción y la segunda indica una progresión del desplazamiento del disco sin reducción para la artritis osteodegenerativa [38].

En este estudio, en el lado derecho se presentó la mayor reciprocidad (22,2\%). El clicking recíproco es un clic en apertura y cierre en el cual el disco se reduce durante la apertura vertical y regresa a su posición desplazada durante el cierre [39]. Del grupo total de estudiantes, el $45,1 \%$ presentó una apertura disminuida, y de los estudiantes con ruidos articulares, el $50 \%$ tenía apertura limitada. Tales porcentajes fueron superiores a lo encontrado en la investigación de Moncada-Escorcia et al. [40], en individuos quienes tenían terceros molares incluidos con $30 \%$ y sin molares incluidos con $28,3 \%$ de apertura $<39 \mathrm{~mm}$. Se aclara que esta fue tomada con una medición de apertura limitada diferente a la de este estudio $(<35 \mathrm{~mm})$. Estos últimos autores exponen que la limitación en apertura puede ser de origen muscular por una contracción mantenida de los músculos elevadores que dificulta la apertura, o de origen meniscal debido a un bloqueo del disco articular. Por otro lado, el estudio de Al-Harthy et al. [41], quienes utilizaron el RDC/TMD, concluyó que 3/39 mujeres con dolor en la articulación presentaron una apertura limitada $\leq 35 \mathrm{~mm}$.

Asimismo, el 39,3\% de los estudiantes tenía dolor a la palpación de los diferentes sitios; y de quienes presentaban ruido articular, el 47,2\% tenía dolor. De igual manera, 33,3\% de estudiantes con ruido articular tenía dolor articular en apertura no asistida y $44,4 \%$, en asistida.

En este sentido, Marklund y Wänman [42], en un estudio de seguimiento a un año de 371 estudiantes de Odontología en Suecia, observaron que el riesgo a desarrollar dolor reportado se incrementó significativamente con la presencia de ruidos articulares (oR 6,9; IC al 95\%: 2,0-23,8), con ruidos articulares clínicamente registrados (OR 11,7; IC al 95\%: 3,3-42,0) y dolor clínicamente registrado (or 6,3; IC al 95\%: 1,5-26,1) en la línea base. A su vez, el riesgo de desarrollar dolor clínicamente registrado se incrementó significativamente con la presencia de ruidos articulares reportados (or 3,6; IC al 95\%: 1,4-9,2) en la línea base. Los ruidos articulares son factor de riesgo potencial para la incidencia de dolor articular, en comparación con aquellos que no tienen ruidos. De alguna forma, este incremento de riesgo contradice la idea de que desarreglos internos de la articulación solo sean una variante de normalidad y no le debería ser dada una atención especial en las valoraciones de riesgo.

Dado lo anterior, la investigación sobre ruidos articulares es muy importante y debe tener en cuenta condiciones como las planteadas en el estudio de Wang et al. [43] sobre la delgadez del disco articular en pacientes con trastornos temporomandibulares (тTM), como dolor orofacial, ruidos articulares o problemas en movimientos mandibulares, en comparación con personas saludables. En esta investigación, se demostró que existe una mayor delgadez de la banda anterior e intermedia en los individuos con TTM, y se concluyó que esta alteración implica tener una carga compresiva disminuida.

Aunque existe poco conocimiento y claridad sobre un tratamiento adecuado para el manejo de TTM, la literatura informa sobre terapias que incluyen cuidados caseros como evitar el exceso de masticación, cambiar la consistencia de la dieta a una más suave, limitar el habla y evitar abrir mucho la boca al bostezar; además, hacer terapia física que incluya aplicación de frío local para dolor agudo o de calor para un bajo grado de dolor crónico [44]. Las anteriores recomendaciones deberían ser de rutina en el manejo de prevención secundaria para el control de los TTM.

De igual forma, Jerjes et al. [45] analizan otras terapias que se recomiendan para el manejo de ттм y sus factores de riesgo, como terapia con placas, el ajuste oclusal, la analgesia y la medicación psicotrópica, y la cirugía; y los tratamientos complementarios como la acupuntura, la terapia con ultrasonido, la estimulación nerviosa eléctrica transcutánea o la electromiografía, que ha sido ampliamente usada en conjunto con relajación y terapia de biofeedback e hipnorrelajación.

Asimismo, se ha estudiado la terapia cognitivacomportamental, la cual permite a los individuos manejar mejor sus dificultades mediante la aplicación de principios de pensamiento y comportamiento que han sido investigados empíricamente. Tales principios se trasladan dentro de estrategias prácticas, las cuales pueden llevar a cambios de pensamiento objetivos y subjetivos, y a estados de comportamiento y sentimientos. Las intervenciones en terapia cognitiva comportamentalincluyen: logros establecidos, desafío de pensamientos negativos automáticos, ejercicios de relajación y respiración, 
ejercicios de visualización cognitiva, estrategias de manejo comportamental, manejo de estrés y habilidades de aserción.

\section{Conclusiones}

La prevalencia de ruidos articulares en jóvenes universitarios es similar a lo reportado por la literatura científica, al igual que los posibles factores de riesgo, entre los que se incluyen algunos aspectos sociodemográficos y de estrés. En relación con las características específicas, se observó una elevada presencia de dolor en pacientes con ruido articular, y cerca de la mitad de ellos presentó una apertura limitada que se ha relacionado con un desplazamiento del disco con reducción.

La investigación en ATM es importante debido a que esta es la estructura que permite la armonía en el sistema estomatognático. El presente estudio es uno de los pocos que se han realizado en países como Colombia que utiliza el RDC/TMD, lo cual facilita comparar la prevalencia de signos que pueden ser indicativos de trastornos de la articulación en individuos de otros países y con antecedentes diferentes.

\section{Agradecimientos}

Carlos Javier López Caicedo, Marley Rodríguez Paredes, Edisson Alexander López, Geovanny Benavides Vallejo, Constanza Díez Cabrera, Marly Bolaños Sánchez y Edwin Omar Bolaños Díaz, por su colaboración en el registro de los ruidos articulares en las diferentes universidades.

\section{Referencias}

[1] Henny FD. Articulación temporomandibular. En: Kruger GO, editor. Cirugía bucomaxilofacial. Buenos Aires: Panamericana; 1986.

[2] American Society of Temporomandibular Joint Surgeons - ASTMJs. Guidelines for Diagnosis and Management of Disorders Involving the Temporomandibular Joint and Related Musculoskeletal Structures. Cranio. 2003;21(1):68-76.

[3] Tanzilli RA, Tallents RH, Katzberg RW, Kyrkanides S, Moss ME. Temporomandibular joint sound evaluation with an electronic device and clinical evaluation. Clin Orthod Res. 2001;4(2):72-8.
[4] Harster-Nadal P. Especulando sobre la apófisis estiloides. Ideas y Trabajos Odontoestomatológicos. 2001;2(2):85-9.

[5] John MT, Hirsch C, Drangsholt MT, Mancl LA, Setz JM. Overbite and Overjet are not Related to Self-Report of Temporomandibular Disorder Symptoms. J Dent Res. 2002;81(3):164-9.

[6] Feteih RM. Signs and Symptoms of Temporomandibular Disorders and Oral Parafunctions in Urban Saudi Arabian Adolescents: A Research Report. Head Face Med. 2006;2:25.

[7] Egermark I, Carlsson GE, Magnusson T. A 20-Year Longitudinal Study of Subjective Symptoms of Temporomandibular Disorders from Childhood to Adulthood. Acta Odontol Scand. 2001;59(1):40-8.

[8] Magnusson T, Egermark I, Carlsson GE. A Prospective Investigation over Two Decades on Signs and Symptoms of Temporomandibular Disorders and Associated Variables: A Final Summary. Acta Odontol Scand. 2005;63(2):99-109.

[9] Grau-León I, Fernández-Lima K, González G, OsorioNúñez M. Algunas consideraciones sobre los trastornos temporomandibulares. Rev Cubana Estomatol. 2005;42(3):1-11.

[10] Oral K, Bal Küçük B, Ebeoğlu B, Dinçer S. Etiology of Temporomandibular Disorder Pain. Agri. 2009;21(3):89-94.

[11] Michelotti A, Cioffi I, Festa P, Scala G, Farella M. Oral Parafunctions as Risk Factors for Diagnostic TMD Subgroups. J Oral Rehabil. 2010;37(3):157-62.

[12] Tallents RH, Macher DJ, Kyrkanides S, Katzberg RW, Moss ME. Prevalence of Missing Posterior Teeth and Intraarticular Temporomandibular Disorders. J Prosthet Dent. 2002;87(1):45-50.

[13] Kanno T, Carlsson GE. A Review of the Shortened Dental Arch Concept Focusing on the Work by the Käyser/ Nijmegen Group. J Oral Rehabil. 2006;33(11):850-62.

[14] Egermark I, Magnusson T, Carlsson GE. A 20-Year Follow-Up of Signs and Symptoms of Temporomandibular Disorders and Malocclusions in Participants with and without Orthodontic Treatment in Childhood. Angle Orthod. 2003;73(2):109-15.

[15] Farella M, Michelotti A, Lodice G, Milani S, Martina R. Unilateral Posterior Crossbite is not Associated with тмJ Clicking in Young Adolescents. J Dent Res. 2007;86(2):137-41.

[16] Ash MM, Ramfjord S. Occlusion. 4. a ed. Philadelphia: Saunders; 1995.

[17] Dibbets JM, Van der Weele LT. Signs and Symptoms of Temporomandibular Disorder (TMD) and 
Craniofacial Form. Am J Orthod Dentofacial Orthop. 1996;110(1):73-8.

[18] González YM. Research Diagnostic Criteria for Temporomandibular Disorders (RDC/TMD Spanish version) Axis I [internet]. s. f. [citado 2009 nov 5]. Disponible en: http://www.rdc-tmdinternational.org/Portals/18/ Translations_RDC/RDC-Spanish.pdf

[19] Uhac I, Kovac Z, Vukovojak S, Zuvic-Butorac M, Grzic R, Delic Z. The Effect of Occlusal Relationships on the Occurrence of Sounds in the Temporomandibular Joint. Coll Antropol. 2002;26(1):285-92.

[20] Soto L, Hernández JA, Villavicencio JE. Trastornos de la articulación temporomandibular en escolares de 5 a 14 años de un centro educativo de Cali. Colomb Med. 2001;32(3):116-20.

[21] Okeson JP. Tratamiento de oclusión y afecciones temporomandibulares. 6. ${ }^{\mathrm{a}}$ ed. Barcelona: Elsevier; 2008.

[22] Tecco S, Crincoli V, Di Bisceglie B, Saccucci M, Macrí M, Polimeni A, Festa F. Signs and Symptoms of Temporomandibular Joint Disorders in Caucasian Children and Adolescents. Cranio. 2011;29(1):71-9.

[23] Johansson A, Unell L, Carlsson GE, Söderfeldt B, Halling A. Gender Difference in Symptoms Related to Temporomandibular Disorders in a Population of 50-Year-Old Subjects. J Orofac Pain. 2003;17(1):29-35.

[24] Wang MQ, Xue F, He JJ, Chen JH, Chen CS, Raustia A. Missing Posterior Teeth and Risk of Temporomandibular Disorders. J Dent Res. 2009;88(10):942-5.

[25] Klobas L, Tegelberg A, Axelsson S. Symptoms and Signs of Temporomandibular Disorders in Individuals with Chronic Whiplash-Associated Disorders. Swed Dent J. 2004;28(1):29-36.

[26] Choi YS, Choung PH, Moon HS, Kim SG. Temporomandibular Disorders in 19-Year-Old Korean Men. J Oral Maxillofac Surg. 2002;60(7):797-803.

[27] Adler NE, Ostrove JM. Socioeconomic Status and Health: What We Know and What We Don't. Ann N Y Acad Sci. 1999;896:3-15.

[28] Elstad JI. How Large Are the Differences-Really? SelfReported Long-Standing Illness among Working Class and Middle Class Men. Sociology of Health \& Illness. 1996;18(4):475-98.

[29] Adler NE, Boyce T, Chesney MA, Cohen S, Folkman S, Kahn RL, Syme SL. Socioeconomic Status and Health. The Challenge of the Gradient. Am Psychol. 1994;49(1):15-24.

[30] Polychronopoulou A, Divaris K. Dental Students' Perceived Sources of Stress: A Multi-Country Study. J Dent Educ. 2009;73(5):631-9.

[31] Shah M, Hasan S, Malik S, Sreeramareddy CT. Perceived Stress, Sources and Severity of Stress among Medical
Undergraduates in a Pakistani Medical School. BMC Med Educ. 2010;10:1-8.

[32] Larrucea C, Castro R, Vera A. Estudio de prevalencia de signos y síntomas de trastornos tempormandibulares (тTM) en un grupo de adultos de Talca, Chile. Rev Dent Chile. 2002;93(3):28-36.

[33] Gesch D, Bernhardt O, Alte D, Schwahn C, Kocher T, John U, et al. Prevalence of Signs and Symptoms of Temporomandibular Disorders in an Urban and Rural German Population: Results of a PopulationBased Study of Health in Pomerania. Quintessence Int. 2004;35(2):143-50.

[34] Michelotti A, Iodice G. The Role of Orthodontics in Temporomandibular Disorders. J Oral Rehabil. 2010;37(6):411-29.

[35] Akana A, Erginb A, Yildirimc M, Öztaşc E. Analysis of Temporomandibular Joint Sounds in Orthodontic Patients. Computers \& Electrical Engineering. 2006;32(4):312-21.

[36] Kirveskari P, Alanen P. Association between Tooth Loss and тмJ Dysfunction. J Oral Rehabil. 1985;12(3):189-94.

[37] Okamoto H, Haraguchi S, Takada K. Laterality of Asymmetry in Movements of the Corners of the Mouth during Voluntary Smile. Angle Orthod. 2010;80(2):223-9.

[38] Ogütcen-Toller M. Sound Analysis of Temporomandibular Joint Internal Derangements with Phonographic Recordings. J Prosthet Dent. 2003;89(3):311-8.

[39] Leader JK, Boston JR, Rudy TE, Greco CM, Zaki HS. The Influence of Mandibular Movements on Joint Sounds in Patients with Temporomandibular Disorders. J Prosthet Dent. 1999;81(2):186-95.

[40] Moncada-Escorcia RA, Martínez-González JM, Merchán-Morales S, Barona-Dorado C, Fernández-Cáliz F. Influencia de los terceros molares retenidos en pacientes con trastornos de la articulación temporomandibular en el hospital de Madrid. Av Odontoestomatol. 2009;25(1):35-40.

[41] Al-Harthy M, Al-Bishri A, Ekberg E, Nilner M. Temporomandibular Disorder Pain in Adult Saudi Arabians Referred for Specialized Dental Treatment. Swed Dent J. 2010;34(3):149-58.

[42] Marklund S, Wänman A. Incidence and Prevalence of Temporomandibular Joint Pain and Dysfunction. A One-Year Prospective Study of University Students. Acta Odontol Scand. 2007;65(2):119-27.

[43] Wang M, Cao H, Ge Y, Widmalm SE. Magnetic Resonance Imaging on TMJ Disc Thickness in TMD Patients: A Pilot Study. J Prosthet Dent. 2009;102(2):89-93.

[44] Selby A. Physiotherapy in the Management of Temporomandibular Disorders. Aust Dent J. 1985;30(4): $273-80$. 
[45] Jerjes W, Upile T, Abbas S, Kafas P, Vourvachis M, Rob J, et al. Muscle Disorders and Dentition-Related Aspects in Temporomandibular Disorders: Controversies in the
Most Commonly Used Treatment Modalities. Int Arch Med. 2008;1(1):1-23. 